Pathology, Liverpool Hospital, Liverpool, NSW, Australia; ${ }^{7}$ Discipline of Pathology, School of Medicine, Western Sydney University, Liverpool, NSW, Australia; ${ }^{8}$ Graduate School of Medicine, University of Wollongong, Wollongong, NSW, Australia

Contact Dr Martin Harb.

E-mail: Martin.Harb@health.nsw.gov.au

1. Heymann WR. Extramammary Paget's disease. Clin Dermatol 1993; 11 $83-7$.

2. Shepherd V, Davidson EJ, Davies-Humphreys J. Extramammary Paget's disease. BJOG 2005; 112: 273-9.

3. Hutchings D, Windon A, Assarzadegan N, Salimian KJ, Voltaggio L, Mongtomery EA. Perianal Paget's disease as spread from non-invasive colorectal adenomas. Histopathology 2021; 78: 276-80.

4. Lam C, Funaro D. Extramammary Paget's disease: summary of current knowledge. Dermatol Clin 2010; 28: 807-26.

5. Liao X, Liu X, Fan X, Lai J, Zhang D. Perianal Paget's disease: a clinicopathological and immunohistochemical study of 13 cases. Diagn Pathol 2020; 15: 1-7.

6. De Nisi MC, D'Amuri A, Toscano M, Lalinga AV, Pirtoli L, Miracco C. Usefulness of CDX2 in the diagnosis of extramammary Paget disease associated with malignancies of intestinal type. Br J Dermatol 2005; 153: 677-9.

7. Ohnishi T, Watanabe S. The use of cytokeratins 7 and 20 in the diagnosis of primary and secondary extramammary Paget's disease. Br J Dermatol 2000; 142: 243-7.

8. Battles OE, Page DL, Johnson JE. Cytokeratins, CEA, and mucin histochemistry in the diagnosis and characterization of extramammary Paget's disease. Am J Clin Pathol 1997; 108: 6-12.

9. Goldblum JR, Hart WR. Perianal Paget's disease: a histologic and immunohistochemical study of 11 cases with and without associated rectal adenocarcinoma. Am J Surg Pathol 1998; 22: 170-9.

DOI: https://doi.org/10.1016/j.pathol.2021.11.011

\section{Adenosquamous carcinoma-like mesonephric adenocarcinoma}

\section{To the Editor,}

Mesonephric adenocarcinoma (MNAC) is a rare malignant tumour derived from remnants of the mesonephric duct in the female genital tract. MNAC usually occurs in the cervix, vagina, uterine body and ovary. The histopathological features of MNAC are the combination of multiple growth patterns, showing glandular, tubular and papillary. Due to the rarity of this disease, its aetiology and precursor lesions remain unclear. All MNAC patients reported thus far underwent surgical resection. Adjuvant radiotherapy and chemotherapy are often used in patients with advanced disease, ${ }^{1}$ but the best scheme and effect of treatment are unknown.

Herein, we present a case of a 57-year-old female with vaginal bleeding, less than the menstrual volume, and no abdominal pain. A magnetic resonance imaging (MRI) scan revealed a $3.2 \mathrm{~cm}$ diameter mass in the right anterior wall of the cervix, with high $\mathrm{T} 1$ and $\mathrm{T} 2$ signals; diffusion weighted imaging (DWI) showed high signal intensity, and the arterial phase was significantly intensified after enhancement. U1trasound imaging revealed an irregular hypoechoic area in the cervix, with uneven internal echo and abundant blood flow signals. The first laboratory workup showed an increased CA125 level of $56.29 \mathrm{U} / \mathrm{mL}$. To confirm the diagnosis, biopsy revealed that the tumour was solid and papillary, with a fibrovascular axis, and mimicked a non-keratinising squamous carcinoma. The lesion was misdiagnosed as squamous cell carcinoma (SCC) due to the morphology of the squamous epithelial growth pattern (morphology mimics a high grade squamous intraepithelial lesion) and the lack of immunohistochemistry. As MRI showed that the tumour invaded the parauterine tissue, a course of neoadjuvant therapy (combination of irinotecan and nedaplatin) was carried out prior to surgery of radical hysterectomy and lymphadenectomy. Before the operation, the level of CA125 was reduced to $37.17 \mathrm{U} / \mathrm{mL}$. Grossly, a $2.9 \times 2.9 \times 2.7 \mathrm{~cm}$ ulcerative mass was located in the cervix, invading the upper third of the vagina. The uterine body, ovaries and fallopian tubes were unremarkable. Microscopically, the mass showed a variety of architectural growth patterns, including papillary (Fig. 1A,B), solid and glandular (Fig. 1C,D) patterns. The pattern of papillary growth had fibrovascular axis, and the morphology was similar to that of papillary SCC. Spindlelike epithelial cells could be seen around the glandular structure; the nucleus was round and oval, and the nucleolus was not obvious. Immunohistochemical studies of the tumour cells yielded the following results: negative for CK5/6 (Fig. 2A), oestrogen receptor (ER), progesterone receptor (PR) and p40; diffusely positive for CD10 (Fig. 2B), GATA3 (Fig. 2D), AE1/AE3 and CK7, and focally positive for P16 (Fig. 2C). No metastasis was found in any of the lymph nodes. The patient received postoperative chemotherapy (combination of irinotecan and nedaplatin) and had no evidence of disease 10 months after the operation.

To the best of our knowledge, this is the first report of adenosquamous carcinoma-like MNAC. Mesonephric duct remnants in the cervix are found in approximately $22 \%$ of adult females. ${ }^{2}$ However, tumours from mesonephric remnants rarely occur in the female reproductive system. The diagnosis of MNAC is difficult and challenging, especially for frozen sections and biopsy specimens. Various morphological patterns can be seen in MNAC, such as glandular, papillary and reticular patterns. The histological features of our case were solid and squamous epithelial growth patterns. Initially, the tumour was misdiagnosed as SCC due to the absence of immunohistochemistry analysis. As MRI showed that the tumour invaded the parauterine tissue, we used next generation sequencing (NGS) technology to detect the paraffin tissue of patients for follow-up treatment. NGS results showed TERT, MSH2, TSC2, ARHGAP6, AURKA, CTNNA2, CTNNA3, FAT3, FGF4, HCK, LRP1, MUC16, NSD1, SLIT2 and SLX4 mutations in the tumour tissue. According to the report of targeted genomic analysis using NGS, most MNACs harbour KRAS mutations, ${ }^{3,4}$ suggesting that $K R A S$ mutations are related to the development of MNAC. The histological features of MNAC with KRAS mutations were adenoid, papillary, cribriform and solid. ${ }^{5}$ Although the morphology of this case also shows tubular, solid and papillary structures, the pattern of papillary growth is similar to papillary SCC. TERT mutations have been reported in $70 \%$ of basal cell carcinomas, $50 \%$ of cutaneous SCC, ${ }^{6} 22 \%$ of cervical SCC and $60 \%$ of oral SCC. ${ }^{7}$ In addition, $80 \%$ of SCC of the urinary bladder ${ }^{8}$ also have TERT mutations. In spindle cell carcinoma and spindle cell or spindle cell/squamous cell differentiated breast carcinoma, TERT mutations are as high as $50 \%$. $^{9}$ It could be concluded that the morphology of this case may be related to TERT mutations.

In summary, cervical MNAC is a rare tumour. Cervical lesions are usually detected by biopsy. Because of the 

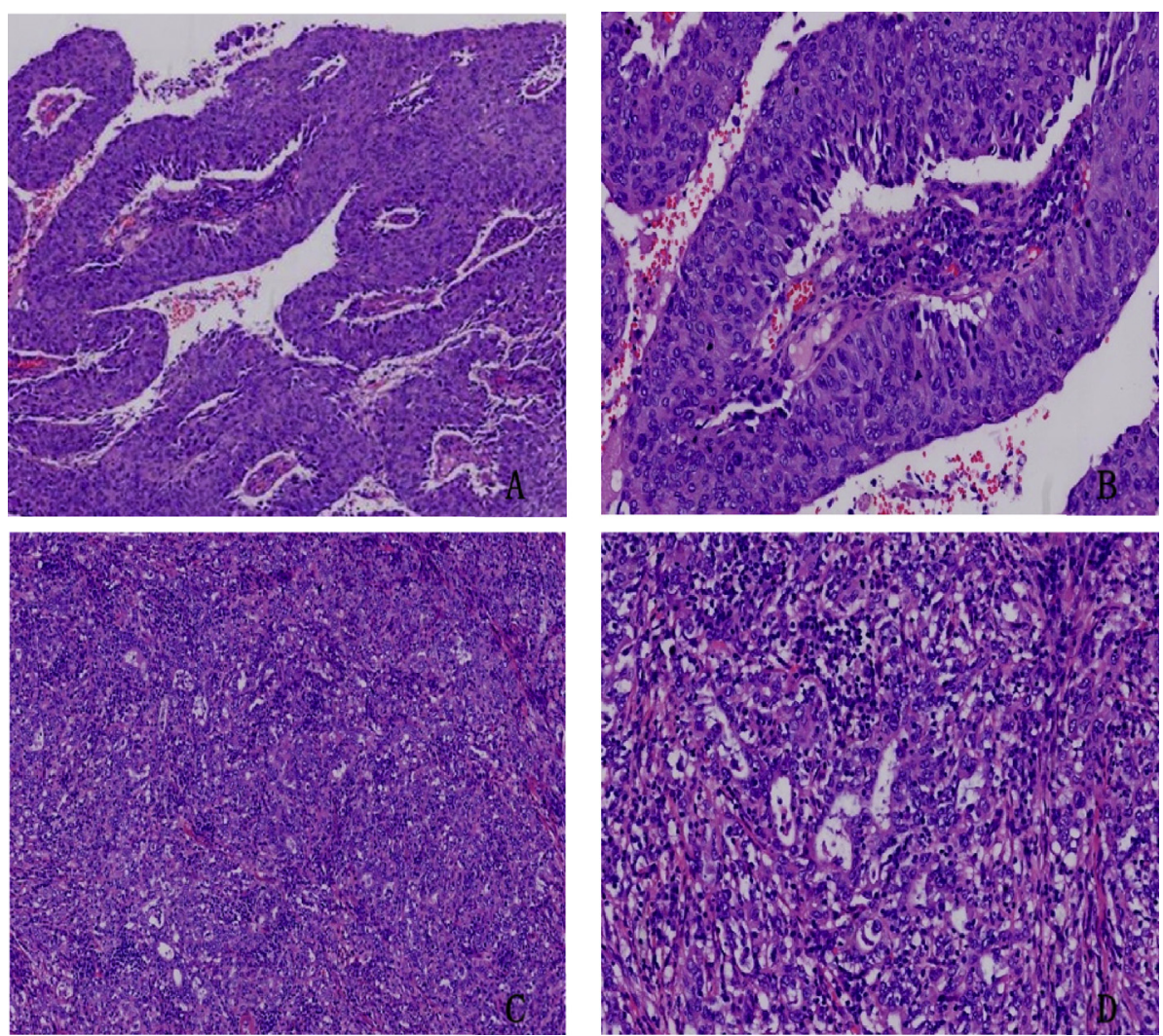

Fig. 1 Histological features of the mesonephric adenocarcinoma: (A,B) the pattern of papillary growth has a vascular axis, and the morphology is similar to that of papillary squamous cell carcinoma; (C,D) the tumour shows a solid growth and glandular patterns.
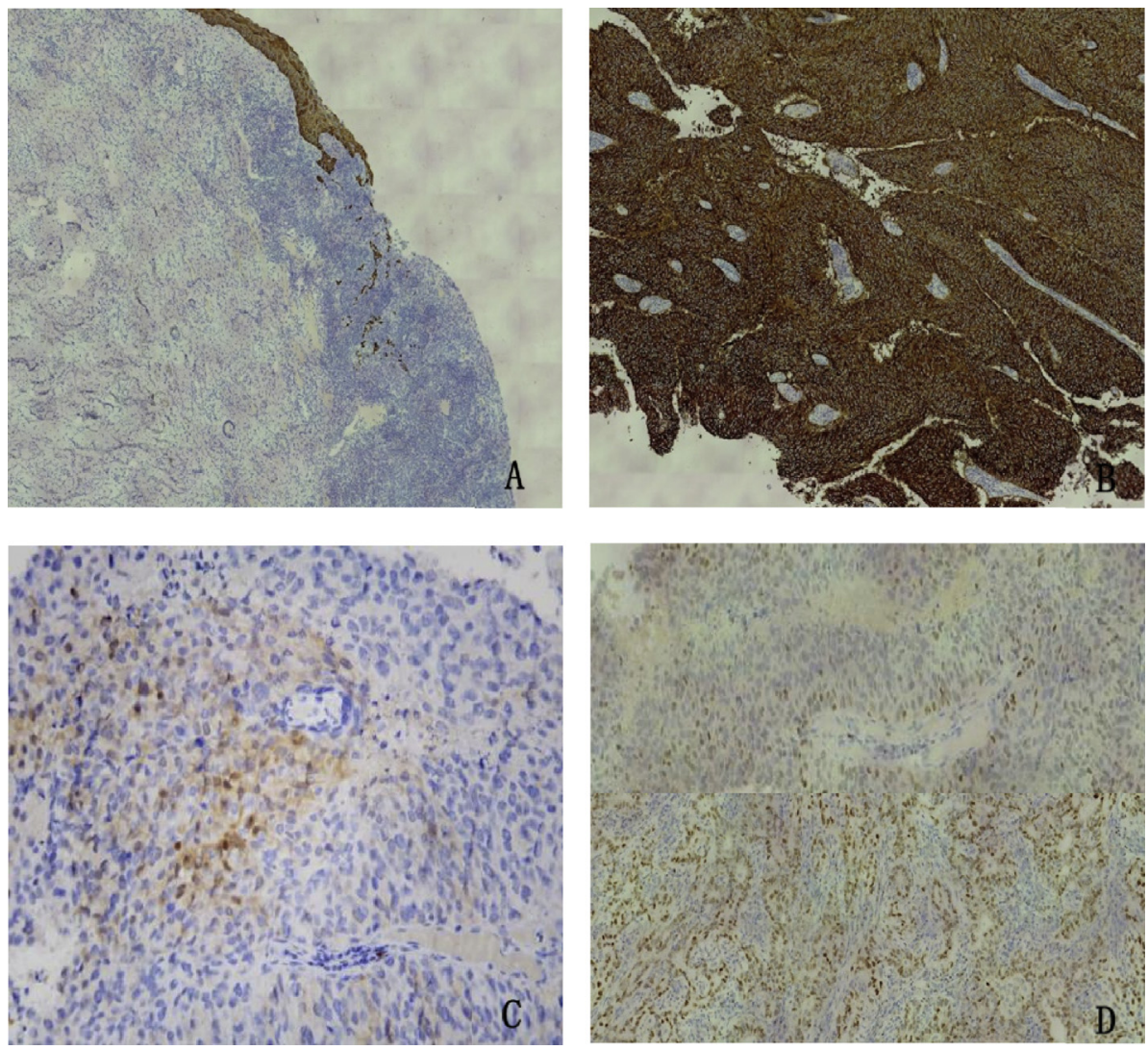

Fig. 2 Immunohistochemical studies of the tumour cells: (A) negative for CK5/6; (B) diffusely positive for CD10; (C) focally positive for P16; (D) diffusely positive for GATA3. 
limitations of biopsy specimens, MNAC is easy to misdiagnose when the morphology is close to that of SCC. When tumours show papillary and squamous epithelial growth patterns by biopsy, it is necessary to carefully observe whether they have adenoid structures and examine them by immunohistochemistry to avoid misdiagnosis.

Conflicts of interest and sources of funding: This work was supported by the Fujian Medical University Qihang Foundation (Grant no. 2018QH1133), the High-Level Hospital Foster Grants from Fujian Provincial Hospital, Fujian Province, China (Grant no. 2019HSJJ25), and the Fujian Provincial Natural Science Foundation Project (Grant no. 2019J01182). The authors state that there are no conflicts of interest to disclose.

\section{Rui Gao ${ }^{1, *}$, Xi Zhang ${ }^{2, *}$, Long Jin ${ }^{1}$}

${ }^{1}$ Department of Pathology, Fujian Provincial Hospital, Provincial Clinical Medical College of Fujian Medical University, Fuzhou, China; ${ }^{2}$ Department of Gastroenterology, Fujian Provincial Hospital, Provincial Clinical Medical College of Fujian Medical University, Fuzhou, China; "Contributed equally as first authors

Contact Prof Long Jin.

E-mail: jlongfjslyy@163.com

1. Dierickx A, Göker M, Braems G, Tummers P, Van den Broecke R Mesonephric adenocarcinoma of the cervix: case report and literature review. Gynecol Oncol Rep 2016; 17: 7-11.

2. Ferry JA, Scully RE. Mesonephric remnants, hyperplasia, and neoplasia in the uterine cervix. A study of 49 cases. Am J Surg Pathol 1990; 14: 1100-11.

3. Na K, Kim HS. Clinicopathologic and molecular characteristics of mesonephric adenocarcinoma arising from the uterine body. Am J Surg Pathol 2019; 43: 12-25.

4. Mirkovic J, Sholl LM, Garcia E, et al. Targeted genomic profiling reveals recurrent KRAS mutations and gain of chromosome $1 \mathrm{q}$ in mesonephric carcinomas of the female genital tract. Mod Pathol 2015; 28: $1504-14$

5. Mirkovic J, McFarland M, Garcia E, et al. Targeted genomic profiling reveals recurrent KRAS mutations in mesonephric-like adenocarcinomas of the female genital tract. Am J Surg Pathol 2018; 42: 227-33.

6. Huang FW, Bielski CM, Rinne ML, et al. TERT promoter mutations and monoallelic activation of TERT in cancer. Oncogenesis 2015; 4: e176.

7. Annunziata C, Pezzuto F, Greggi S, et al. Distinct profiles of TERT promoter mutations and telomerase expression in head and neck cancer and cervical carcinoma. Int J Cancer 2018; 143: 1153-61.

8. Cowan M, Springer S, Nguyen D, et al. High prevalence of TERT promoter mutations in primary squamous cell carcinoma of the urinary bladder. Mod Pathol 2016; 29: 511-5.

9. Krings G, Chen YY. Genomic profiling of metaplastic breast carcinomas reveals genetic heterogeneity and relationship to ductal carcinoma. $\operatorname{Mod}$ Pathol 2018; 31: 1661-74.

DOI: https://doi.org/10.1016/j.pathol.2021.11.012

\section{Phosphoglyceride crystals deposition disease: a rare pseudosarcomatous growth with peculiar predilections}

To the Editor,

Phosphoglyceride crystals deposition disease (PCDD) is an exceedingly rare disease with striking resemblance to sarcoma radiologically and clinically. There have been only 13 reported cases so far. Interestingly, all these cases were exclusively reported from Japan (Table 1). There is no apparent reason for this peculiar country predilection. There are no gender predilection, congenital abnormalities, metabolic disorders, or familial history associated with the disease. The affected patients ranged from 37 to 76 years of age. Our case illustrates Malaysia's first reported case of PCDD.

Herein we report a case of a 69-year-old Malay man, who presented with a slow growing mass over the medial aspect of his right calf for the previous $4-5$ years. He recalled a traumatic laceration 10 years ago by a bamboo stalk at the same site. His blood uric acid level was found to be normal (325 $\mu \mathrm{mol} / \mathrm{L}$ ). Radiological findings were suggestive of a peripheral nerve sheath tumour; however, malignancy could not be excluded because there was no clear evidence of fat plane demarcation with the gastrocnemius and soleus muscles (Fig. 1). Thus, a wide excision was performed with part of the soleus muscle, paratenon of Achilles tendon and periosteum removed. Intraoperative features also revealed a malignant appearing mass measuring $10 \mathrm{~cm}$ in diameter with necrotic tissue anterior to the mass, and adherence to the adjacent fascia and periosteum.

Macroscopically, the mass was lobulated and appeared confined to the subcutaneous layer. It had a soft tan to yellowish cut surface with haemorrhagic areas. Microscopically, the mass was well circumscribed by a thick fibrous capsule, containing lobules of foreign body granuloma. These lobules were separated by fibrous septae of varying thickness. The foreign body granulomas were composed of numerous round eosinophilic fibrillary crystals, surrounded and engulfed by sheets of foreign body type multinucleated giant cells (Fig. 2). These crystals showed spectacular corona-shaped string-like reflective particles when examined under polarised light. The crystals and surrounding macrophages showed negative periodic acid-Schiff (PAS) staining, while CD68 immunohistochemistry showed retained staining within the macrophage's cytoplasm and membrane. The patient was discharged well and given long-term followup at his nearest local clinic.

The diagnosis of PCDD is mainly based on the characteristic histological features as seen in our patient's microscopy findings. Occasional asteroid bodies may also be seen. The crystals typically do not dissolve during the usual formalin fixation and paraffin embedding process, and they are usually so soft that thin sections could be performed without decalcification.

Miura et al. ${ }^{1}$ in 2000 reported that these crystals show diffuse, strong, purple-black staining with gold hydroxamic acid method and focal weak staining can be seen with von Kossa's method and Berlin blue stain.

The actual aetiology of PCDD remains unknown; however, there is an association with previous injury in all cases. Initially, PCDD was attributed to a discontinued drug containing vitamin E, named Nibenal, that was in use in Japan from the year 1950-1960. The drug was made from ethanolic extraction of bovine liver. It was used repeatedly in bilateral arm intramuscular injections by a patient in the Miura et ll. $^{1}$ paper as treatment for chilblain. She later developed bilateral brachial muscle PCDD. However, this was proven to be not related as subsequent reported patients with PCDD were not exposed to the drug. ${ }^{2}$ 